See discussions, stats, and author profiles for this publication at: https://www.researchgate.net/publication/329521774

\title{
Feed-forward and Feedback Control in Astrocytes for Ca2+-based Molecular Communications Nanonetworks
}

Article in IEEE/ACM Transactions on Computational Biology and Bioinformatics · December 2018

DOI: $10.1109 / \mathrm{TCBB} .2018 .2887222$

CITATIONS

3

2 authors, including:

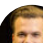

Michael Barros

Tampere University

62 PUBLICATIONS 204 CITATIONS

SEE PROFILE

Some of the authors of this publication are also working on these related projects:

Project $\quad$ Cybersecurity in Brain-Computer Interfaces $(\mathrm{BCI})$ View project

Project Application of Control Theory in Molecular Communication for the Treatment of Alzheimer's Disease View project
READS

75 


\title{
Feed-forward and Feedback Control in Astrocytes for $\mathrm{Ca}^{2+}$-based Molecular Communications Nanonetworks
}

\author{
Michael Taynnan Barros, Member, IEEE, Subhrakanti Dey, Senior Member, IEEE,
}

\begin{abstract}
Synaptic plasticity depends on the gliotransmitters' concentration in the synaptic channel. And, an abnormal concentration of gliotransmitters is linked to neurodegenerative diseases, including Alzheimer's, Parkinson's, and epilepsy. In this paper, a theoretical investigation of the cause of the abnormal concentration of gliotransmitters and how to achieve its control is presented through a $\mathrm{Ca}^{2+}$-signalling-based molecular communications framework. A feed-forward and feedback control technique is used to manipulate $\mathrm{IP}_{3}$ values to stabilise the concentration of $\mathrm{Ca}^{2+}$ inside the astrocytes. The theoretical analysis of the given model aims i) to stabilize the $\mathrm{Ca}^{2+}$ concentration around a particular desired level in order to prevent abnormal gliotransmitters' concentration (extremely high or low concentration can result in neurodegeneration), ii) to improve the molecular communication performance that utilises $\mathrm{Ca}^{2+}$ signalling, and maintain gliotransmitters' regulation remotely. It shows that the refractory periods from $\mathrm{Ca}^{2+}$ can be maintained to lower the noise propagation resulting in smaller time-slots for bit transmission, which can also improve the delay and gain performances. The proposed approach can potentially lead to novel nanomedicine solutions for the treatment of neurodegenerative diseases, where a combination of nanotechnology and gene therapy approaches can be used to elicit the regulated $\mathbf{C a}^{2+}$ signalling in astrocytes, ultimately improving neuronal activity.
\end{abstract}

Index Terms-Molecular Communication, Tripartite Synapses, $\mathrm{Ca}^{2+}$ Signalling, Astrocytes, Control Theory.

\section{INTRODUCTION}

The field of biological and medical science has witnessed, in recent years, the impact of multidisciplinary research efforts that utilise engineering theories, materials and technologies. Examples of this impact include new approaches for nanomedicine [1], [2], smart drug delivery systems [3] and optogenetics [4], which has seen the fields of nanobiotechnology and information technology being brought together. Telecommunication engineers now are investigating biological communication processes that can either be used to understand the biological signalling processes or develop artificial communication systems at the nanoscale. The latter research topic is known as Molecular Communication [5], and its potential applications include sensor and actuator nanonetworks for the human body, as well as new forms of environmental monitoring for smart cities [6].

Inside the human brain, there is a plurality of communication systems with an interplay of different domains such

M. T. Barros is with the Telecommunication Software \& Systems Group (TSGS), Waterford Institute of Technology (WIT), Ireland.

S. Dey is with Inst of Telecomm Research, University of South Australia, Adelaide, Australia.

E-mail: mbarros@tssg.org, Subhra.Dey@unisa.edu.au as the electro-chemical synapses, involving multi-scalability in time and space. This phenomenon has attracted massive attention from the recently-formed molecular communication community due to the many communication issues present within neuronal signalling, neuronal networks and cortical circuits. On the other hand, the historically studied bipartite synapse (neuron-to-neuron) is now accepted as the tripartite synapses, which are formed by a three-way communication of a pre-synaptic neuron, an astrocyte and a post-synaptic neuron [7] [8]. The internal $\mathrm{Ca}^{2+}$ concentration can be influenced by post-synaptic voltage and the communication between astrocytes inside a network of cells and affect the gliotransmitter concentration in the synaptic channel [9]. Gliotransmitters are chemicals released from astrocytes and triggered by their $\mathrm{Ca}^{2+}$ signals, which serve as information carriers in the tripartite synapses, and one such gliotransmitter is glutamate [10]. Through the Glutamate Dependent NMDA Receptors $(G N M D A R)$, the astrocytes play a significant role in numerous brain processes such as plasticity, learning and memory processes [11]. Therefore stable regulation of $\mathrm{Ca}^{2+}$ signalling in astrocytes and their communication is critical. Communication problems in the tripartite synapses can lead to serious diseases, including Alzheimer's, epilepsy, schizophrenia, Parkinson's and depression [12]. The modelling of these communication processes and the application of control theoretic methods for $\mathrm{Ca}^{2+}$ signalling have been previously suggested as a prospect for prevention of neurodegenerative diseases [13].

To this end, a control theoretic model to achieve stable levels of intracellular $\mathrm{Ca}^{2+}$ signalling is proposed for deliberately influencing the gliotransmitter concentration indirectly and remotely inside astrocyte communications nanonetworks. A theoretical framework is developed based on a feed-forward and feedback control methodology, to maintain stable astrocytes' cytosolic $\mathrm{Ca}^{2+}$ concentration. Since proteins are easier to stimulate, control of $\mathrm{IP}_{3}$ is used as a regulation mechanism for intracellular $\mathrm{Ca}^{2+}$ signalling [14]. This framework opened the doors for further contributions including:

- Regulation of $\mathrm{Ca}^{2+}$ Signalling in Astrocytes - A mathematical framework is developed to calculate the desired $\mathrm{Ca}^{2+}$ levels based on a given desired $\mathrm{IP}_{3}$ value and also the stability of the system (the system in this context is the point of communication between the astrocyte and neuron inter-cellular signalling). Finally, a disturbance analysis is used to investigate the benefits of having a feed-forward 


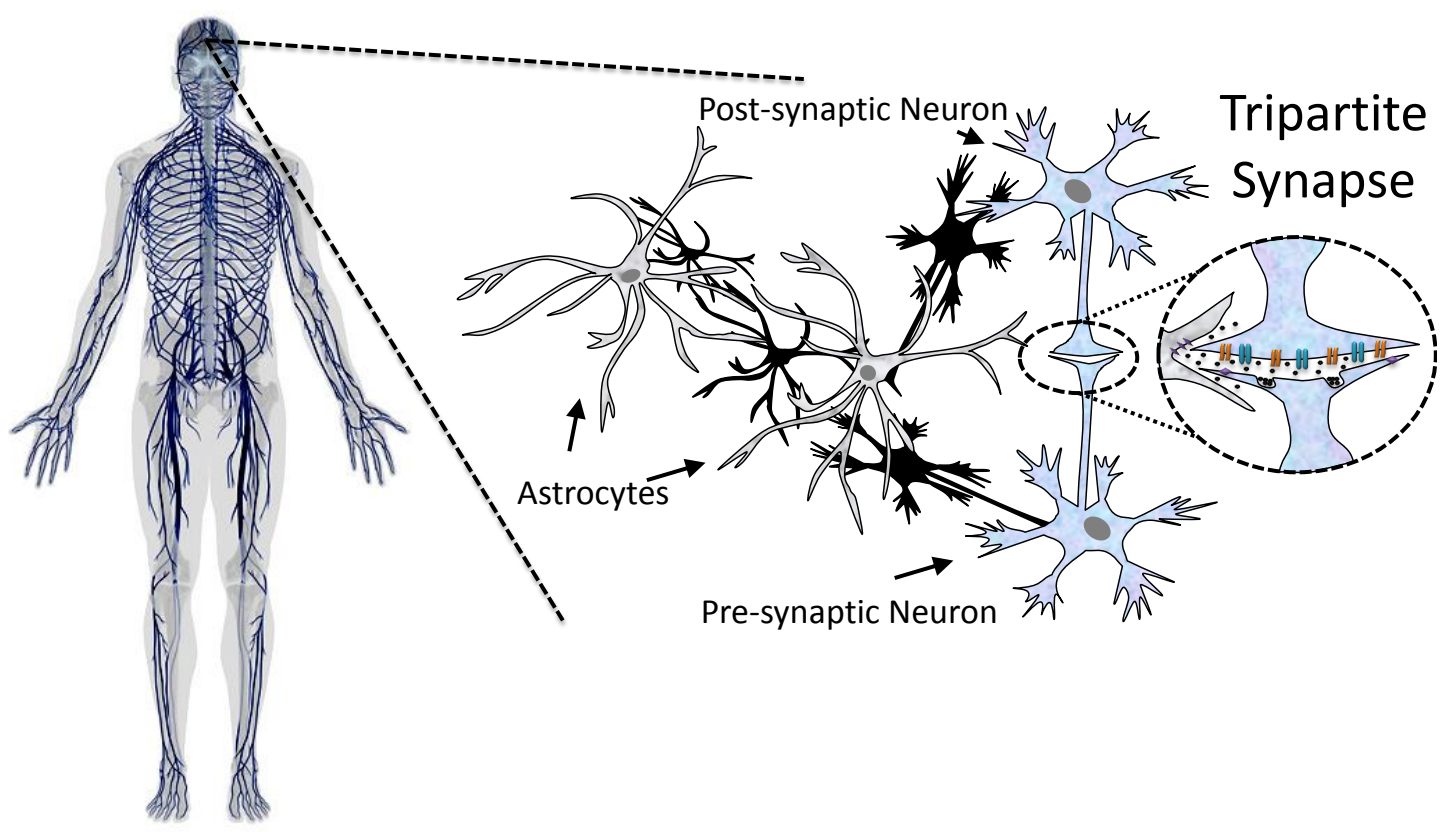

Fig. 1: Tripartite synapses overview, showing the communication process between the astrocyte cell and the pre-synaptic neuron, as well as the post-synaptic neuron. The three-way communication process emits gliotranmitters which are molecules important for maintaining synaptic transmission quality.

component in the control design. The proposed technique achieves the control of $\mathrm{Ca}^{2+}$ levels in the cytosol of astrocytes with proven stability.

- Improved Performance of Molecular Communication System - The use of $\mathrm{Ca}^{2+}$ signalling for molecular communication can result in a high quantity of noise propagating through the tissue, resulting in low data rates. However, the control theoretic method proposed in this paper regulates the $\mathrm{Ca}^{2+}$ levels, resulting in a minimum amount of noise, which in turn achieves superior communication performance with increased the data rate, improved gain and decreased delay.

The paper is organised as follows. $\S I$ introduces the tripartite synapses and the intracellular $\mathrm{Ca}^{2+}$ signalling model for astrocytes. §III presents the oscillation behaviour of regular $\mathrm{Ca}^{2+}$ signalling process and the problem statement. §IV presents the feed-forward and feedback control technique for astrocytes' cytosolic $\mathrm{Ca}^{2+}$ concentration regulation followed by a stability analysis. $\S \mathrm{V}$ presents the application of the control function in the $\mathrm{Ca}^{2+}$-signalling-based molecular communication system. §VI presents the numerical results and analysis of the application of the control technique for elimination of $\mathrm{Ca}^{2+}$ signalling oscillations, disturbance, data rate, molecular gain and molecular delay improvements. §VII presents a discussion about the future envisioned applications. Finally, §VIII concludes the paper.

\section{TRIPARTITE SYNAPSES}

Fig. 1 shows an accurate illustration of the tripartite synapses. The concentration of gliotransmitters in the region connecting both the neurons and the astrocyte is important for synapses' plasticity. Researchers have been able to identify the importance of the astrocytes in the tripartite synapses [8], and the communication process is illustrated in Fig. 2. In the tripartite synapses, the stimulation of the $\mathrm{IP}_{3}$ production in the astrocytes will initiate the $\mathrm{Ca}^{2+}$ signalling process. This stimulus starts either from the post-synaptic, pre-synaptic and other astrocytes in the form of gliotransmitters or adenosine triphosphate (ATP). The increased $\mathrm{IP}_{3}$ values triggers the release of $\mathrm{Ca}^{2+}$ into the cytosol from the endoplasmic reticulum. High quantity of $\mathrm{Ca}^{2+}$ then provoke the release of glutamate ${ }^{1}$ into the synaptic channel. Glutamate is also released from the pre-synaptic neuron invoking an increase of $\mathrm{Ca}^{2+}$ concentration in the astrocytes. These glutamate molecules go back to the pre-synaptic terminal either to inhibit or assist further glutamate release. Therefore, the intracellular $\mathrm{Ca}^{2+}$ signalling in astrocytes of the tripartite synapses dynamically regulates synaptic transmission. It is still debatable also the influence of different types of neurons on the signalling in astrocytes, and the absence of the modulatory activity from pre-synaptic neurons and interneurons [15]. Therefore, we initially neglect these effects for the sake of defining an initial scenario whereby control can be achieved. Based on this, the focus is on the core of this sequential communication process by concentrating on the intracellular $\mathrm{Ca}^{2+}$ signalling process in astrocytes.

The intracellular $\mathrm{Ca}^{2+}$ signalling model in astrocytes consists of state equations for the $\mathrm{Ca}^{2+}$ concentration in the cytosol $(C)$ (1), kinetics of $\mathrm{IP}_{3}$ receptors $(h)(2)$, as well as the $\mathrm{IP}_{3}$ concentration (I) (3). This model is proposed in [16], and a visual illustration of the model is presented in Fig. 3. The reason behind the choice of this model is twofold:

\footnotetext{
${ }^{1}$ One type of gliotransmitter, which activates certain signalling processes in the cells.
} 


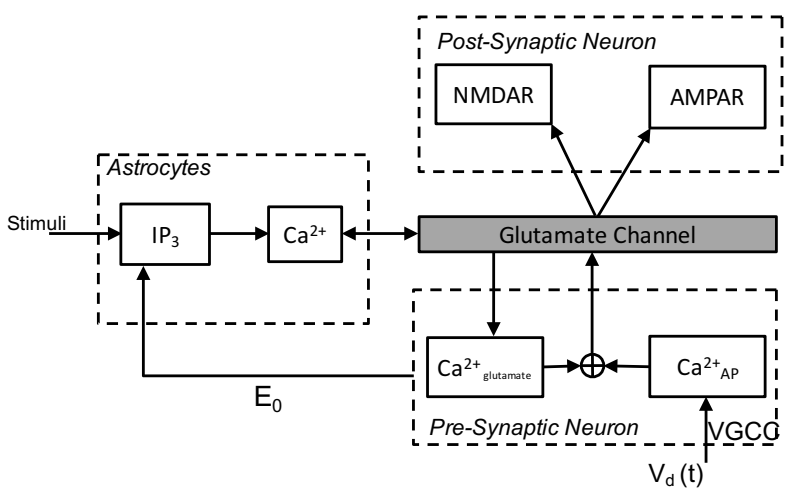

Fig. 2: Model for the Tripartite Synapses. Pre-synaptic neuron, postsynaptic neuron and the astrocyte communicates through a gliotransmitter channel that is invoked from $\mathrm{Ca}^{2+}$ signalling.

i) it is simple and accurate enough to enable the creation of a low complexity control model, and ii) it has been validated through experiments with astrocytes. The main state equations are defined as follows:

$$
\begin{gathered}
\frac{d C}{d t}=\sigma_{1}+\sigma_{2}-\sigma_{3}, \\
\frac{d h}{d t}=\frac{H-h}{\tau}, \\
\frac{d I}{d t}=\frac{1}{\alpha}\left(i_{0}-I\right)+\beta \mathcal{H}\left(E_{0}-35\right)
\end{gathered}
$$

where $\alpha$ is the constant degradation time of $\mathrm{IP}_{3}$ concentration, $i_{0}$ is the $\mathrm{IP}_{3}$ concentration in equilibrium, $\beta$ is the production rate of $\mathrm{IP}_{3}$ ions, $E_{0}$ is the pre-synaptic potential and $\mathcal{H}($.$) is$ the Heaviside function. $\mathrm{Ca}^{2+}$-induced $\mathrm{Ca}^{2+}$ release $(\mathrm{CICR})$ is the trigger process of $\mathrm{Ca}^{2+}$ from the sarco(endo)plasmic reticulum by existing $\mathrm{Ca}^{2+}$ within the cytosol. The quantities $H, \tau$ are defined in (8), (9) and (10). The function $\sigma_{1}$ models the $C I C R$ and is defined as:

$$
\sigma_{1}=v m^{3} h^{3}\left[c_{0}-\left(1+C_{1}\right) C\right]
$$

where $v$ is the maximal CICR rate, $c_{0}$ is the total cell-free $\mathrm{Ca}^{2+}$ concentration depending on the cytosol volume, and $C_{1}$ is the ratio between the cytosol and endoplasmic reticulum volume.

The $\mathrm{IP}_{3}$ and $\mathrm{Ca}^{2+}$ binding process that is responsible for providing stable $\mathrm{IP}_{3}$ kinetics is represented as:

$$
m=\left(\frac{I}{I+d}\right)\left(\frac{C}{C+d_{3}}\right)
$$

where $d$ is the $\mathrm{IP}_{3}$ dissociation constant and $d_{3}$ is the $\mathrm{Ca}^{2+}$ activation-dissociation constant.

The function $\sigma_{2}$ denotes the leakage of $\mathrm{Ca}^{2+}$ to the cytosol from the sarco(endo)plasmic reticulum and is represented as:

$$
\sigma_{2}=v_{1}\left[c_{0}-\left(1+C_{1}\right) C\right]
$$

where $v_{1}$ is the maximal rate of $\mathrm{Ca}^{2+}$ leakage from the endoplasmic reticulum.

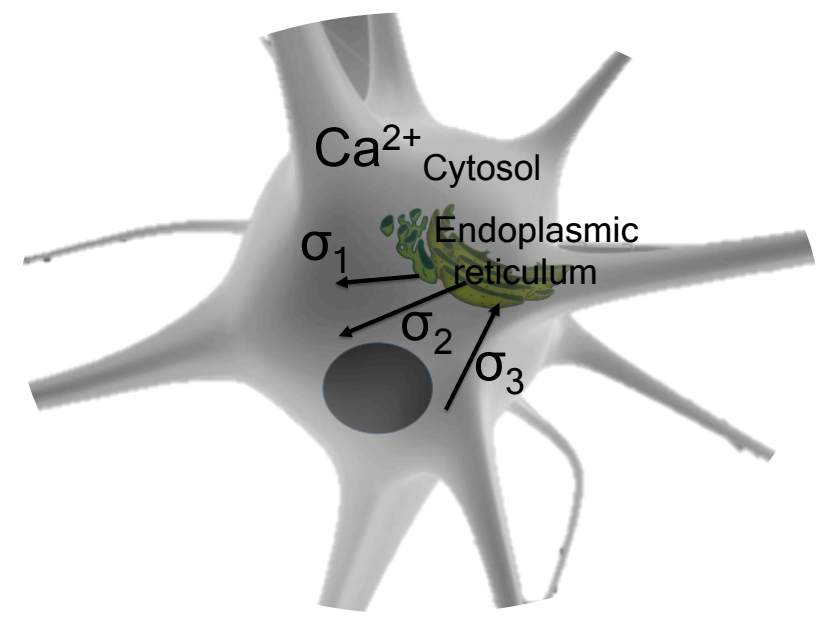

Fig. 3: Intracellular $\mathrm{Ca}^{2+}$ signalling model for astrocytes. The flux/efflux rates control the concentration of $\mathrm{Ca}^{2+}$ signalling. The function $\sigma_{1}$ models the $\mathrm{Ca}^{2+}$-induced $\mathrm{Ca}^{2+}$ release $(C I C R)$. The function $\sigma_{2}$ denotes the leakage of $\mathrm{Ca}^{2+}$ from the endoplasmic reticulum to the cytosol, and the function $\sigma_{3}$ denotes denotes the SERCA-driven $\mathrm{Ca}^{2+}$ uptake from the cytosol into the endoplasmic reticulum.

The efflux of $\mathrm{Ca}^{2+}$ from the sarco(endo)plasmic reticulum to the endoplasmic reticulum (SERCA) is represented as:

$$
\sigma_{3}=\frac{v_{2} C^{2}}{k^{2}+C^{2}}
$$

where $v_{2}$ is the maximal rate of SERCA uptake, and $k$ is $\mathrm{Ca}^{2+}$ binding affinity.

The following equations are important for modelling $h$ :

$$
\begin{gathered}
H=\frac{Q}{Q+C} \\
\tau=\frac{1}{a(Q+C)} \\
Q=\frac{I+d}{I+d_{2}} d_{1}
\end{gathered}
$$

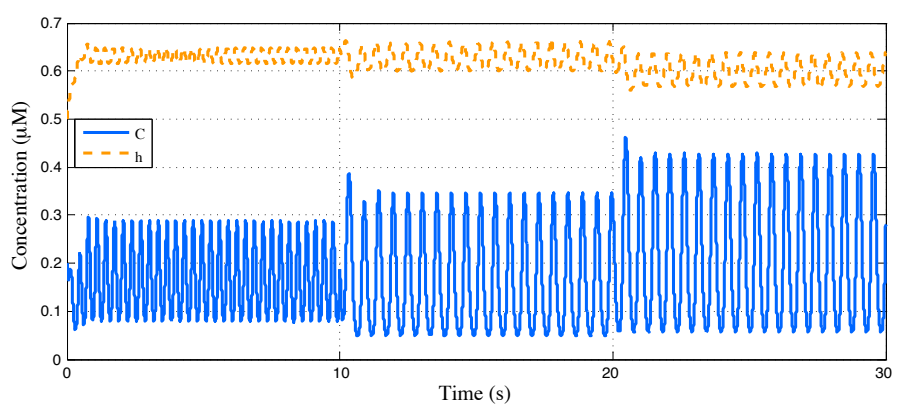

Fig. 4: $\mathrm{Ca}^{2+}$ oscillation with respect to time. In this illustration the $\mathrm{IP}_{3}=0.5 \mu \mathrm{M}$. The $\mathrm{Ca}^{2+}$ concentration $(C$ - blue line $)$ oscillates alongside with the kinetics of $\mathrm{IP}_{3}$ receptors ( $h$ - dashed yellow line). The signal amplitude modulation (when $k=0.1 \mu M$ ) and frequency modulation (when $k=0.05 \mu M$ ) show the different variations that $\mathrm{Ca}^{2+}$ oscillations can take. 
where $d_{1}$ is the $\mathrm{Ca}^{2+}$ inactivation dissociation constant, $d_{2}$ is the $\mathrm{IP}_{3}$ dissociation constant and $a$ is the $\mathrm{IP}_{3}$ receptors binding rate for $\mathrm{Ca}^{2+}$ inhibition.

\section{Problem Statement}

Neurodegenerative diseases are related to the quality of the synapses (plasticity) in neuronal communication. The poor concentration of glutamate inside the synaptic channel will lead to poor propagation in the synapses, causing lack of memory, insomnia, depression which are symptoms of most neurodegenerative diseases. Current treatment techniques for neurodegeneration are based on drugs that are not efficient and only helps to eliminate symptoms and not treat them. In the tripartite synapses, neurodegeneration is directly linked to signal plasticity in the synaptic channel and the amount of glutamate in there. Proteins and amino-acids can interact with both neurons and astrocytes causing abnormal levels of glutamate, which is mostly caused by the main generation process, the intracellular astrocyte $\mathrm{Ca}^{2+}$ signalling. Achieving $\mathrm{Ca}^{2+}$ regulation in astrocytes can indirectly control the glutamate release and potentially improve the synaptic plasticity. Control of $\mathrm{Ca}^{2+}$ has been theoretically achieved in our previous work [17]. The primary challenge now is to provide an analysis on the astrocytes $\mathrm{Ca}^{2+}$ concentration of the tripartite synapses and, therefore, create a theoretical framework that supports the indirect control of glutamate.

For this, the problem of controlling levels of $\mathrm{Ca}^{2+}$ in the cytosol is investigated in this paper and its influence on an astrocyte network communication. More specifically, internal $\mathrm{Ca}^{2+}$ signalling is characterised by oscillations invoked by a particular range of $\mathrm{IP}_{3}$. Fig. 4 shows the $\mathrm{Ca}^{2+}$ oscillation at $\mathrm{IP}_{3}=0.5 \mu \mathrm{M}$ and when production rate of $\mathrm{IP}_{3}(\beta-$ Eq. 3$)$ varies from 0.1-1.5 $\mu \mathrm{M} / \mathrm{s}$ and modulates both the amplitude (when $k=0.1 \mu M, \mathrm{M}$ for mols) and frequency (when $k=0.05 \mu M)$ of the signal. The shape of the signal follows both the $\mathrm{IP}_{3}$ kinetics as well as the binding affinity of $\mathrm{Ca}^{2+}$ released from the endoplasmic reticulum (7). Also, because of the $\mathrm{Ca}^{2+}$ pool dependency $(C)$, the signal shape is additionally affected by the intracellular regulation mechanisms including pre-synaptic signals.
Fig. 5 shows how the $\mathrm{IP}_{3}$ can affect the intracellular $\mathrm{Ca}^{2+}$ signalling based on the pre-synaptic voltage influence $\left(E_{0}\right)$, by varying the $\mathrm{IP}_{3}$ concentration and showing its effects over time in three different cases: on the left $E_{0}=50 \mathrm{mV}$, at the center $E_{0}=35 \mathrm{mV}$ and on the right $E_{0}=0 \mathrm{mV}$. An increase of $\mathrm{IP}_{3}$ in the system occurs when the $\mathrm{IP}_{3}$ is activated and when $E_{0}>0 \mathrm{~V}$. Since $\beta$, the factor contributing to the increase of $\mathrm{IP}_{3}$ based on $E_{0}$ is the biggest influence on the internal $\mathrm{Ca}^{2+}$ concentration (3), its control is highly critical for the regulation of $\mathrm{Ca}^{2+}$ concentration levels. Another indication of this is evidenced by the quick decrease of the $\mathrm{Ca}^{2+}$ concentration value once $\mathrm{IP}_{3}$ achieves a constant level. In addition, since the synapses happen periodically, we assume that there are only activated neurons and there is no inter-synaptic interference in the synaptic channel from the post-synaptic dynamic release of glutamate. The $\mathrm{IP}_{3}$ is, in conclusion, a decisive factor for $\mathrm{Ca}^{2+}$ regulation, in which its increase is controlled by $\beta$. We further explore this property with a mathematical model that enables the intracellular $\mathrm{Ca}^{2+}$ signalling control by regulating $\mathrm{IP}_{3}$ levels.

Regulation can potentially be achieved in two ways: the total elimination of rapid oscillatory behaviour of $\mathrm{Ca}^{2+}$ waves or the regulation of these oscillations. The oscillatory elimination can be less complex and offers a way for future treatment of neurodegeneration, where periods of stables levels of $\mathrm{Ca}^{2+}$ can be realized as a regulatory mechanism for achieving a regenerated activity of the tripartite synapses as well as its plasticity [16]. Moreover, keeping $\mathrm{Ca}^{2+}$ at stable levels as a method for periodic regulation is appealing as a countermeasure against noise, since random addition or removal of $\mathrm{Ca}^{2+}$ by various ways can be detrimental to this system especially when $\mathrm{IP}_{3}$ varies from $0.1-0.3 \mu \mathrm{M}$ or $0.7-0.9 \mu \mathrm{M}$ [16]. It is also beneficial to regulate the oscillatory behaviour of $\mathrm{Ca}^{2+}$ according to a desired oscillatory pattern. Our objectives, then, will comprise of both these types of regulation.

\section{Feed-Forward and Feedback Control of INTRACELlular $\mathrm{CA}^{2+}$ Signalling IN Astrocytes}

Since $\mathrm{IP}_{3}$ activation can only be performed in highly controlled settings [17], its regulation of $\mathrm{Ca}^{2+}$ concentration is more suitable for in-vitro scenarios at the moment. The


Fig. 5: Effect of both $\mathrm{IP}_{3}$ and the $E_{0}$ on the astrocyte intracellular $\mathrm{Ca}^{2+}$ concentration, $E_{0}=50 \mathrm{mV}$ (left), $E_{0}=35 \mathrm{mV}$ (center) and $E_{0}=0 \mathrm{mV}$ (right). The $\mathrm{Ca}^{2+}$ concentration is highly dependent on the increasing factor of $\mathrm{IP}_{3}$, specifically when it is activated. This means that $\mathrm{IP}_{3}$ stability can become an important factor in indirectly controlling the astrocyte intracellular $\mathrm{Ca}^{2+}$ concentration. 


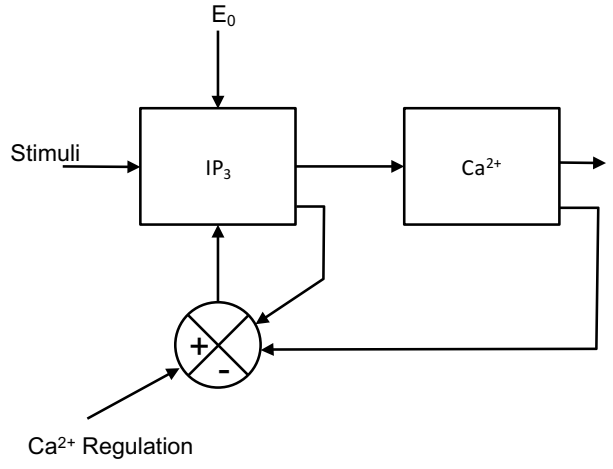

Fig. 6: $\mathrm{Ca}^{2+}$ regulation flowchart based on the regulation of cytosolic $\mathrm{IP}_{3}$. The input rate of $\mathrm{IP}_{3}$ is a function of the current cytosolic $\mathrm{Ca}^{2+}$ and the current $\mathrm{IP}_{3}$ levels, which makes it both a state feedback and feed-forward control.

proposed approach can be potentially realised with the help of nanoparticles or silicon devices inside a cell to inhibit or activate $\mathrm{Ca}^{2+}$ as part of the control model.

Fig. 6 shows a functional block diagram of the desired $\mathrm{Ca}^{2+}$ signalling set point regulation. Before proceeding further, assume for the rest of the paper that the intracellular $\mathrm{IP}_{3}$ channel of the astrocyte is constantly activated, i.e. $\mathcal{H}\left(E_{0}-35\right)=1$. Note that for other values of the Heaviside function (such as $\left.\mathcal{H}\left(E_{0}-35\right)=\frac{1}{2}\right)$, a similar analysis can be applied. By denoting the column vector state $\mathbf{x}=\left[\begin{array}{lll}C & h & I\end{array}\right]^{\prime}$, rewrite (1), (2) and (3) as the following nonlinear controlled state space system

$$
\frac{d \mathbf{x}}{d t}=f(\mathbf{x})+\mathbf{B} u
$$

where $f(\mathbf{x})=\left[f_{1}(\mathbf{x}) f_{2}(\mathbf{x}) f_{3}(\mathbf{x})\right]^{\prime}$, and $f_{1}()=.\sigma_{1}+\sigma_{2}+$ $\sigma_{3}, f_{2}()=.\frac{H-h}{\tau}$, and $f_{3}()=.\frac{1}{\alpha}\left(i_{0}-I\right)$, and $\mathbf{B}=\left[\begin{array}{lll}0 & 0 & 1\end{array}\right]^{\prime}$. The control variable $u$ represents the state feedback and feedforward based $\mathrm{IP}_{3}$ regulation parameter given by (see also [14]):

$$
\beta=\beta_{f}-K_{f}\left(C-C_{f}\right),
$$

where $\beta_{f}$ is the feed-forward control representing the desired $\mathrm{IP}_{3}$ level $I_{f}, C_{f}$ is the associated desired $\mathrm{Ca}^{2+}$ concentration level and $K_{f}$ is the linear feedback gain. Note that although not visible in the above equation, there is an associated value of $h$ as well, which is denoted by $h_{f}$. Denote the entire associated state vector as $\mathbf{x}_{\mathbf{f}}=\left[\begin{array}{lll}C_{f} & h_{f} & I_{f}\end{array}\right]^{\prime}$. Then it follows that

$$
\frac{d \mathbf{x}_{\mathbf{f}}}{d t}=f\left(\mathbf{x}_{\mathbf{f}}\right)+\mathbf{B} u_{f}
$$

where of course, $u_{f}=\beta_{f}$. Here, the measured output is the $\mathrm{Ca}^{2+}$ concentration level, so that at the desired level, the output is given by $\bar{h}\left(\mathbf{x}_{\mathbf{f}}\right)=C_{f}$.

Remark 1: Note that without loss of generality, one can assume that in the uncontrolled case, i.e., when $\beta=0$, there is an equilibrium point at the origin for the nonlinear dynamical system $\frac{d \mathbf{x}}{d t}=f(\mathbf{x})$ [14], implying $f(\mathbf{0})=0$. This fact will be used in the stability analysis of the system when the control law (12) is applied in a subsequent subsection.

A similar control law to (12) including the feed-forward term was proposed for disturbance rejection in roll-to-roll manufacturing system [14]. In this model, no specific disturbance variables are implemented, which may be present in cases of diseases that may affect the normal regulated $\mathrm{Ca}^{2+}$ concentration levels. However, since the nature of the problem investigated in this paper is the elimination of $\mathrm{Ca}^{2+}$ oscillations, it is clear that not only such a control function perfectly fits into our modelling of the nonlinear controlled $\mathrm{Ca}^{2+}$ regulation system, but also allows us the flexibility of including disturbance factors in future modelling extensions.

\section{A. $\beta_{f}$ and $C_{f}$ Relationship}

To obtain the required regulation factor $\beta_{f}$ for a desired $C_{f}$, a mathematical relationship between $\beta_{f}$ and $C_{f}$ is obtained as follows. Suppose there is an equilibrium point (in the controlled case when $\beta>0)$ at $\left(\begin{array}{lll}h^{o} & c^{o} & I_{0}\end{array}\right)$, then $\frac{d h}{d t}=0$ at $h=h^{o}$ and $\frac{d C}{d t}=0$ at $C=c^{o}$, and $\frac{d I}{d t}=0$ for $I=I_{0}$. Rewriting Eq. 1 and Eq. 2, obtaining:

$$
\begin{gathered}
\left(v_{1}+z_{o}\left(h^{o}\right)^{3}\right) \times\left(C_{0}-\left(1+C_{1}\right) c^{o}\right)=\frac{v_{2}\left(c^{o}\right)^{2}}{k^{2}+\left(c^{o}\right)^{2}}, \\
h^{o}=\frac{Q_{o}}{Q_{o}+c^{o}},
\end{gathered}
$$

where

$$
z_{o}=v\left(\frac{I_{o}}{I_{o}+d}\right)^{3}\left(\frac{c^{o}}{c^{o}+d_{3}}\right)^{3},
$$

and

$$
Q_{o}=\frac{I_{o}+d}{I_{o}+d_{2}} d_{1} .
$$

Here $I_{o}$ is obtained from for Eq. 3, (since $\mathcal{H}\left(E_{0}-35\right)=1$ as previously assumed), by solving:

$$
\frac{d I}{d t}=\left(\frac{i_{0}}{\alpha}+\beta\right)-\frac{I}{\alpha} \text {. }
$$

which yields

$$
I=\left(i_{0}+\alpha \beta\right)\left(1-e^{\frac{-t}{\alpha}}\right) .
$$

As $t \rightarrow \infty, I$ becomes a constant $I_{o}$, which is represented as:

$$
I_{o}=\left(i_{0}+\alpha \beta\right) .
$$

Substituting the above value of $I_{O}$ in the equations for $Q_{o}, h^{o}$ and $z_{o}$, one can solve the nonlinear equation (14) to obtain $c^{o}=C_{f}$ for a given $I_{o}=\beta_{f}$.

\section{B. System Stability}

Since anomalies in the dynamics of $\mathrm{Ca}^{2+}$ signalling can lead to diseases and also tissue death, a stabilising control model for such a system is critically required. In the following, a stability analysis is presented that ensures that how the feed-forward and feedback based regulation of the $\mathrm{IP}_{3}$ level can maintain the $\mathrm{Ca}^{2+}$ level very close to its desired value. This requires a linearisation of the nonlinear dynamics around the equilibrium using Taylor's series expansion, assuming one initialises the system very near the origin. Recall from Remark 1 that is considered without loss of generality that there is an equilibrium at the root of the uncontrolled model.

Note that by carrying out a Taylor's expansion of (11) around the origin, and ignoring the higher order nonlinear 
terms (since the expansion is obtained very close to the origin) one obtains

$$
\frac{d \mathbf{x}}{d t}=\mathbf{A}(\mathbf{x})+\mathbf{B}\left(\beta_{f}-\left[\begin{array}{lll}
K_{f} & 0 & 0
\end{array}\right]\left[\begin{array}{c}
C-C_{f} \\
h-h_{f} \\
I-I_{f}
\end{array}\right]\right)
$$

where $\mathbf{A}(\mathbf{x})$ is the linear part of $f(\mathbf{x})$. Note that $\mathbf{A}$ is given by

$$
\mathbf{A}=\left[\begin{array}{lll}
\frac{\partial f_{1}}{\partial C} & \frac{\partial f_{2}}{\partial C} & \frac{\partial f_{3}}{\partial C} \\
\frac{\partial f_{1}}{\partial h} & \frac{\partial f_{2}}{\partial h} & \frac{\partial f_{3}}{\partial h} \\
\frac{\partial f_{1}}{\partial I} & \frac{\partial f_{2}}{\partial I} & \frac{\partial f_{3}}{\partial I}
\end{array}\right]_{\mid C=0, h=0, I=0}
$$

It follows easily that

$$
\mathbf{A}=\left[\begin{array}{ccc}
-v_{1}\left(1+C_{1}\right) & 0 & 0 \\
0 & -a \times \frac{d d_{1}}{d_{2}} & a \times \frac{d_{1}\left(d_{2}-d\right)}{d_{2}^{2}} \\
0 & 0 & -\frac{1}{\alpha}
\end{array}\right]
$$

One can also show from linearization of (13) around the origin that the following is true:

$$
\frac{d \mathbf{x}_{\mathbf{f}}}{d t}=\mathbf{A}\left(\mathbf{x}_{\mathbf{f}}\right)+\mathbf{B} \beta_{f}
$$

Defining $\zeta=\mathbf{x}-\mathbf{x}_{\mathbf{f}}$, having (by subtracting (24) from (21))

$$
\frac{d \zeta}{d t}=(\mathbf{A}-\mathbf{B K}) \zeta
$$

where $\mathbf{K}=\left[\begin{array}{lll}K_{f} & 0 & 0\end{array}\right]$. It follows then that the matrix $\mathbf{A}-\mathbf{B K}$ is given by

$$
\mathbf{A}-\mathbf{B K}=\left[\begin{array}{ccc}
-v_{1}\left(1+C_{1}\right) & 0 & 0 \\
0 & -a \times \frac{d d_{1}}{d_{2}} & a \times \frac{d_{1}\left(d_{2}-d\right)}{d_{2}^{2}} \\
-K_{f} & 0 & -\frac{1}{\alpha}
\end{array}\right]
$$

Finally, the eigenvalues of $\mathbf{A}-\mathbf{B K}$ can be easily shown to be $\left(-v_{1}\left(1+C_{1}\right),-a \times \frac{d d_{1}}{d_{2}},-\frac{1}{\alpha}\right)$. Since all of these eigenvalues are negative, the matrix $\mathbf{A}-\mathbf{B K}$ is Hurwitz, which implies that $\mathbf{x}(t)-\mathbf{x}_{\mathbf{f}}(t) \rightarrow 0$ as $t \rightarrow \infty$, or $C \rightarrow C_{f}$ as $t \rightarrow \infty$. This proves that under the feed-forward and feedback control law proposed in this work, the $\mathrm{Ca}^{2+}$ level can be maintained at the desired level after a sufficiently large time interval. Simulation studies will show that the convergence of $C$ to the desired value $C_{f}$ is considerably fast.

\section{V. $\mathrm{CA}^{2+}$-SIGNALling-BASED MoleCUlaR COMMUNiCATION SYSTEM MODEL}

In this section, a single mathematical framework merges the $\mathrm{Ca}^{2+}$-signalling, control and synthetic biology models alongside diffusion and 3D cellular tissue modelling. Barros et. al. has extensively studied this model [18], [19], [20]. Spatio-temporal dynamics is captured by a 3D cellular tissue model, correlating phenomena and variables at different scales and analysing how the control technique will perform. The description is composed of two parts: the 3D modelling of the cellular tissues, and the stochastic model for the scheduling of reactions within individual cells.

\section{A. 3D Modelling of Cellular Tissue Structure}

Consider a cellular tissue space $(S)$ composed of $I \times J \times K$ cells $(c)$, where $c_{i, j, k}(i=1 \ldots I ; j=1, \ldots J$ and $k=$ $1, \ldots K)$ denotes an arbitrary cell in the tissue. The cells are connected with a maximum of six neighbouring cells. The topological organisation of the astrocytes is considered following regular specifications in [21]. A Stochastic Solver computes the values of each pool over time, selecting and executing scheduled reactions. The pool is negatively or positively affected by a constant $\alpha$ when a particular reaction is run. More details on the astrocytes' pools and reactions can be found in Section II.

Modelling diffusion in a cellular tissue area captures the temporal-spatial dynamics of intercellular $\mathrm{Ca}^{2+}$ signaling. The model considers $\mathrm{Ca}^{2+}$ concentration difference for temporalspatial characteristic as follows:

$$
Z_{\Delta}(i, j, k, n, m, l)=\frac{D}{v}\left(\left|Z_{n, m, l}-Z_{i, j, k}\right|\right) \times p_{(.)}
$$

where $n \in(i-1, i+1), m \in(j-1, j+1), l \in(k-1, k+1), D$ is the diffusion coefficient, $v$ is the volume of the cell, and $Z_{\Delta}$ is the difference in $\mathrm{Ca}^{2+}$ concentration between the cells. $p_{(.)}$ is the probability of the gap junction opening. More details of this model can be found in [19].

At each time step, the Gillespie algorithm [22] (a Stochastic Solver) schedules a particular astrocyte $\in S$, its reaction $(R)$ and the time duration of this particular reaction $(t)$, therefore determining the quantity of each pool over time. Each reaction is allocated a reaction constant $\left(a_{r}\right)$. Considering that $\alpha_{0}$ is the summation of all $a_{r}$ in $R$, the next reaction chosen $r_{u}$ will be:

$$
r_{u}=\operatorname{MAX}\left\{\frac{a_{r_{j}}}{\alpha_{0}}=\frac{a_{r_{j}}}{\sum_{j=1}^{|R|} a_{r_{j}}}\right\}, u \in \mathbb{N}, u \in R
$$

which follows the roulette wheel selection process, by choosing the events based on their probability values. However, $u$ must satisfy the following restriction:

$$
\sum_{j=1}^{u-1} \frac{\alpha_{r_{j}}}{\alpha_{0}}<\rho_{2} \leq \sum_{j=1}^{u} \frac{\alpha_{r_{j}}}{\alpha_{0}}
$$

in which $\rho_{2}$ is a uniform random variable with values in the range $(0,1)$.

At each time step $(t)$, a time lapse $\left(\tau_{t}\right)$ is derived based on $\alpha_{0}$, and is represented as:

$$
\alpha_{0} \cdot \tau_{t}=\ln \frac{1}{\rho_{1}}
$$

in which $\rho_{1}$ is a uniform random variable with values in the range $(0,1)$. This process ends when $\sum_{i=0}^{|T|} \tau_{i}<t_{\theta}$, where $T$ is the set of $t$ and $t_{\theta}$ is the maximum simulation time.

\section{B. System Design}

Our objective here is to model the communication system between a stimulation point inside an astrocyte network and a receiver cell. The stimulation point is envisioned as the 
location of the control input inside the network, and the quantification of the impact of controlling $\mathrm{Ca}^{2+}$ a few cells away from the desired location is needed. This can assess how the control technique can be achieved remotely within a range of micrometres. This scenario is depicted in Fig. 7.

A block diagram of the system is shown in Fig. 8. The communication system is composed of a transmitter, channel and receiver. The details of each system component are described in the following.

1) Transmitter: The transmitter is comprised of an encoder, an equaliser and a digital modulator. The stimuli will initiate the $\mathrm{Ca}^{2+}$ production based on a bit sequence acting as the message. We use equal probabilities of $0 \mathrm{~s}$ and $1 \mathrm{~s}$ in the message. The stimuli can be either mechanical, chemical or electrical in the astrocyte case. However, we will assume ideal electrical stimuli since it has a quicker cellular response. The equaliser will guarantee signal shape at ideal levels based on the control function we are proposing; this is explained in Section V-B4. The modulation process is based on ON-OFF Keying, where the transmitter releases $\mathrm{Ca}^{2+}$ when a bit 1 is to be sent or no $\mathrm{Ca}^{2+}$ emission for bit 0 .

2) Channel: The channel is comprised of the propagation medium of $\mathrm{Ca}^{2+}$ signals in the tissue. After its release point, the signal is subject to internal $\mathrm{Ca}^{2+}$ regulation processes in each cell along the path to the receiver end. This is shown in Fig 8, where the astrocyte intracellular signalling is modelled as in Sec.II, which include the following reactions: stimulation, amplification, storage, release and diffusion.

Another impact on signal propagation along the tissue is the cell-to-cell connections. These are modelled by the 3D tissue modelling found in the previous section and the physical cellular connection to its neighbours, termed gap junctions. We assume each cell has a total of six gap-junctions that connect to each adjacent cell. Detailed gap junction models have been explored in [23], [19]. The model considers two states of conductance for each gap junction from each cell: State $H H$ : Both gates are in a high conductance state. This probability is denoted by $p_{H H}$; State $H L$ : One gate is in a high conductance state, and the other is in a low conductance state. This probability is denoted by $p_{H L} ;$ State $L H$ : One gate is in a low conductance state, and the other is in a high conductance state. This probability is denoted by $p_{L H}$; State $L L$ : Both gates are in a low conductance state. This probability is denoted by $p_{L L}$. These probabilities should obviously satisfy $p_{H H}+p_{H L}+p_{L H}+p_{L L}=1$. The probability distribution is obtained from [19].

3) Receiver: The Receiver is comprised of three essential elements: the equaliser, the digital demodulator and the decoder. Based on the upcoming $\mathrm{Ca}^{2+}$ to the cellular cytosol, the equaliser will attempt to reshape the signal using the control technique. This process is explained in more detail in Section V-B4. The OOK demodulator, which is a low pass filter, can regenerate the signal in a digital format and then bits are detected through a concentration based detection technique [24].

4) Equalizer: The equalization process is realized based on the control function developed in Section IV. Based on the input signal, the signal equalisation is fixed based on the existing production of $\mathrm{IP}_{3}$ (12). In the transmitter, this is aligned with cellular stimuli for message encoding. In the receiver, the upcoming $\mathrm{Ca}^{2+}$ concentration will trigger the production of $\mathrm{IP}_{3}$ in the cell, re-initiating the oscillatory response.

The toggle switch is then responsible for activating the feed-forward feedback control and thereby re-shaping the signal. The theoretical two-gene bistable toggle switch has been proposed by Gardner et al [25]. The switch consists of two constitutive promoters (coloured black and grey) and two repressor genes (also coloured black and grey). The black repressor protein silences the grey promoter, which drives production of the grey repressor protein. Conversely, the grey repressor protein silences the black promoter, which drives production of the black repressor protein. Thus, if the black repressor protein were produced, the grey repressor protein could not be produced, and vice versa. This is an example of a synthetic bistable toggle switch similar to the theoretical one in Figure 9. The presented approach is simply a possible method of activating a dependent stimulus of $\mathrm{IP}_{3}$ production based on current $\mathrm{Ca}^{2+}$ levels. It is noteworthy that based on the dependency of the equaliser to incoming $\mathrm{Ca}^{2+}$ signal, this mechanism only serves as part of the equaliser and it is not used as a noise controller.

5) Assumptions: Both the transmitter and the receiver have been engineered with the proposed control function and its toggle switch activation in the form of an equaliser. In this way, both devices are capable of ideally responding to a desired level of $\mathrm{IP}_{3}$ and, thereby maintaining stable levels of $\mathrm{Ca}^{2+}$ concentration during the transmission period $T_{b}$. The toggle switch is activated with the two following processes: 1) the external cellular stimuli in the transmitter and 2) the upcoming $\mathrm{Ca}^{2+}$ signals in the receiver. This process is depicted in Fig. 8. The system design also has the following assumptions.

Assumption 1: Synchronization between the transmitter and receiver is considered in ideal settings [18].

Assumption 2: The effects of the synthetic circuit on the stimulus of $\mathrm{IP}_{3}$ are also considered ideal. Note that this work only concentrates on the effect of the $\mathrm{Ca}^{2+}$ control in the astrocyte communication.

Assumption 3: The used modulation is On-Off Shifting Keying. We do not extend the analysis to other types of modulation.

\section{Performance AnAlysis}

The performance analysis of the proposed regulation of $\mathrm{Ca}^{2+}$ concentration levels for astrocytes is provided below. The discussion is divided into four parts for a proper understanding of the system under controllable conditions. First, how the control system eliminates intracellular $\mathrm{Ca}^{2+}$ oscillations in astrocytes is shown by solving the problem defined in Section III. This is followed by a disturbance analysis, where Gaussian noise is applied to the intracellular $\mathrm{Ca}^{2+}$ signalling in order to add an abnormal behaviour to the system. Here we observe the effectiveness of the control strategy with and without the the feed-forward part of the control. Finally, performance improvement in the $\mathrm{Ca}^{2+}$-signalling- 


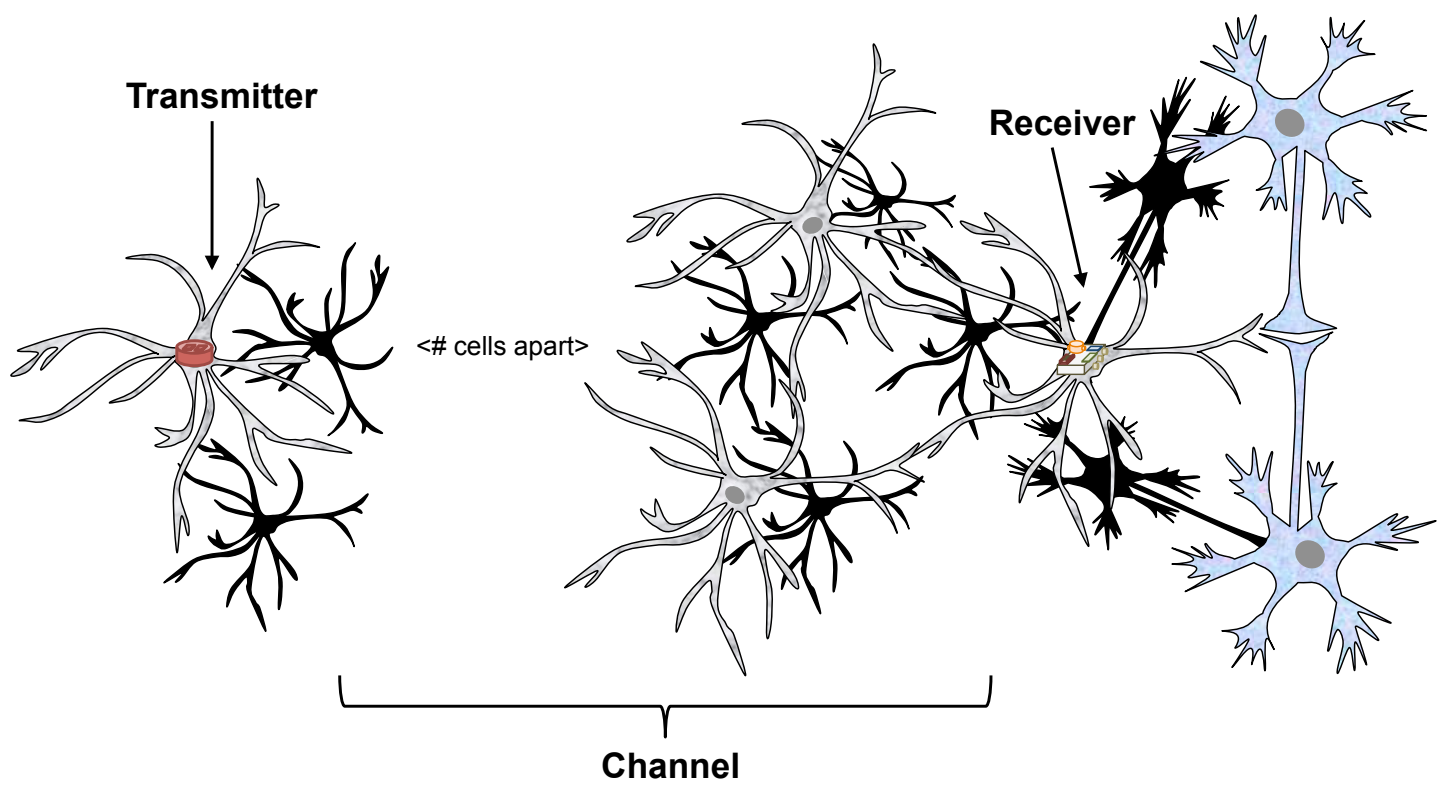

Fig. 7: Astrocyte network communication system. The synthetic transmitter and receiver are placed inside a astrocyte cellular tissue. However, the receiver is always in interface with other two neurons forming a tripartite synapses.

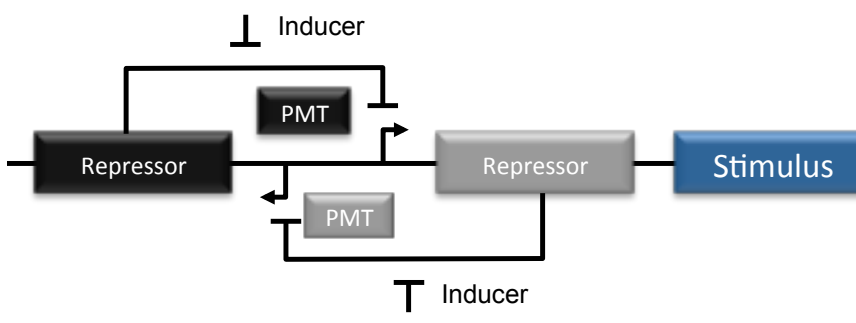

Fig. 9: The black gene "repressor" is transcribed from its black promoter (PMT). The black repressor protein binds to the grey promoter to block the production of the grey repressor protein. The grey repressor protein blocks the production of the black repressor protein when the grey repressor protein binds to the black promoter. To detect which state the toggle switch is in, Stimulus was placed downstream of the grey promoter so that the grey repressor and stimulus are produced simultaneously. Once the stimulus is produced, the $\mathrm{IP}_{3}$ production starts as well.

based molecular communication system of astrocyte networks as a result of the control action is shown.
1) Regulation of Intracellular $\mathrm{Ca}^{2+}$ Oscillations : As mentioned in Section III, a complete elimination of the intracellular $\mathrm{Ca}^{2+}$ oscillation is one of the desired objectives of the control strategy. To this end, (1), (2) and (3) are solved using the parameter values found in Table I. For the control technique, $\beta$ in (3) is replaced by (12), in order to integrate the feed-forward and feedback control elements to the system. A value of $C_{f}=0.32 \mu M$ is chosen, which is a central value of the system, and this leads to the desired value of $\beta_{f}$ with an appropriate calibration of the $K_{f}$.

A total elimination of the $\mathrm{Ca}^{2+}$ oscillation is obtained using the proposed mechanism, and this is illustrated in Fig. 10. Eq. 12, which represents the state feedback and feed-forward control, can efficiently adjust $\beta$ accordingly and maintain $\mathrm{Ca}^{2+}$ concentration levels throughout the period shown across all $\mathrm{Ca}^{2+}$ signal modulation variations. This positive result demonstrates the effectiveness and potential of utilising the control technique to stabilise the excessive $\mathrm{Ca}^{2+}$ concentration that may either lead to neurodegenerative diseases or artificial molecular communication, which will be further explored in

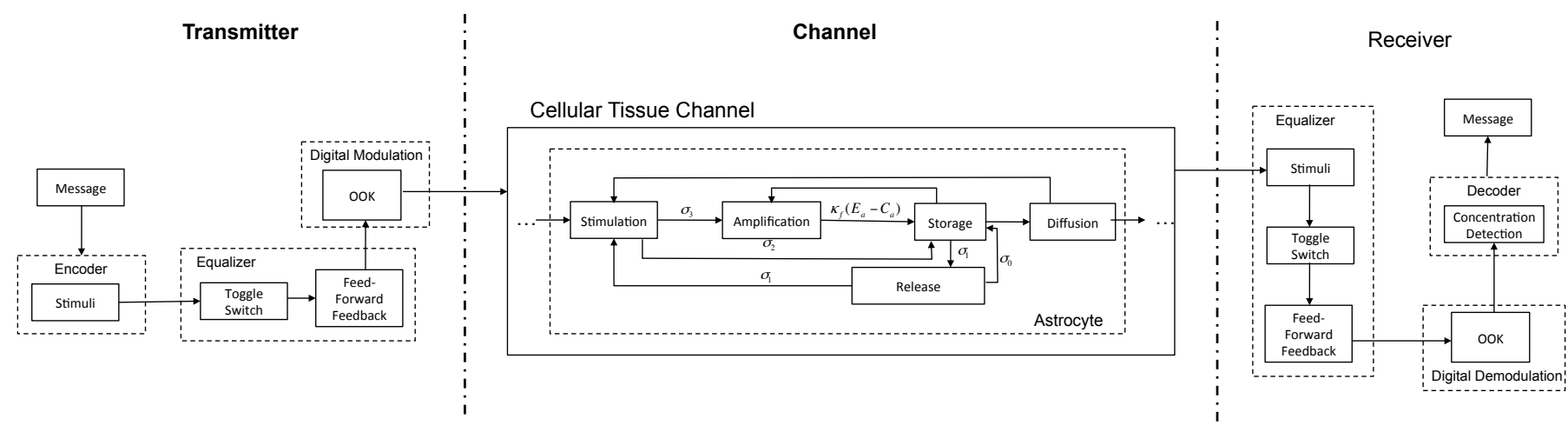

Fig. 8: System design. The transmitter has an equalizer to inhibit intracellular noise in the generated cellular signalling. The channel is represented by a cellular tissue. The receiver is also equipped with an equalizer to regenerate the signal affected by noise at different levels. 
TABLE I: Simulation parameters for astrocytes. [26]

\begin{tabular}{cc}
\hline Variable & Value \\
\hline$v$ & $6 \mathrm{~s}^{-1}$ \\
$v_{1}$ & $0.11 \mathrm{~s}^{-1}$ \\
$c_{0}$ & $2.0 \mu M$ \\
$C_{1}$ & 0.185 \\
$v_{2}$ & $0.9 \mathrm{Ms}^{-1}$ \\
$k$ & $0.1 \mu M$ \\
$d$ & $0.13 \mu M$ \\
$d_{1}$ & $1.049 \mathrm{~s}^{-1}$ \\
$d_{2}$ & $0.9434 \mu M / s$ \\
$d_{3}$ & $0.08234 \mu M$ \\
$a$ & $0.2 \mathrm{~s}^{-1}$ \\
$\alpha$ & $1 / 0.00014 \mu M$ \\
$i_{0}$ & $0.160 \mu M$ \\
$\beta$ & $0.1-1.5 \mu M$ \\
$E_{0}$ & $35 \mathrm{mV}$ \\
\hline
\end{tabular}

the following subsections. Further results with a different set of scenarios are presented in [17].

With an increased level of control complexity, we also achieved regulated oscillatory control of intracellular $\mathrm{Ca}^{2+}$ concentration, see Fig. 11. Compared to the pre-synaptic influence replicated from [27], with a $4 \mathrm{~Hz}$ firing of the presynaptic neuron, regulated oscillatory control can be achieved by a continuous change of $C_{f}$ (12). We considered $C_{f}=$ $0.5(\sin (2 \pi 56)+1.01)$ obtained from a non-linear regression of $C$ values. Even though the results are satisfactory, this type of control is consequently harder to implement in experimental settings due to the oscillatory behaviour that $C_{f}$ has to have. This requires more complex implementation systems that use synthetic biology, and consequently, cellular reprogramming techniques using synthetic DNA that will have computation capabilities allowing both monitoring and actuation in the cellular stimuli based on oscillatory functions.

2) Analysis of System Disturbance: Feed-forward control techniques are used in control theory to stabilise systems under the presence of disturbances. The presented $\mathrm{Ca}^{2+}$ signalling model so far does not include any disturbances or noise components. To analyse the benefit of the feedback control technique with and without feed-forward separately, a noise component is integrated and determine the effectiveness of

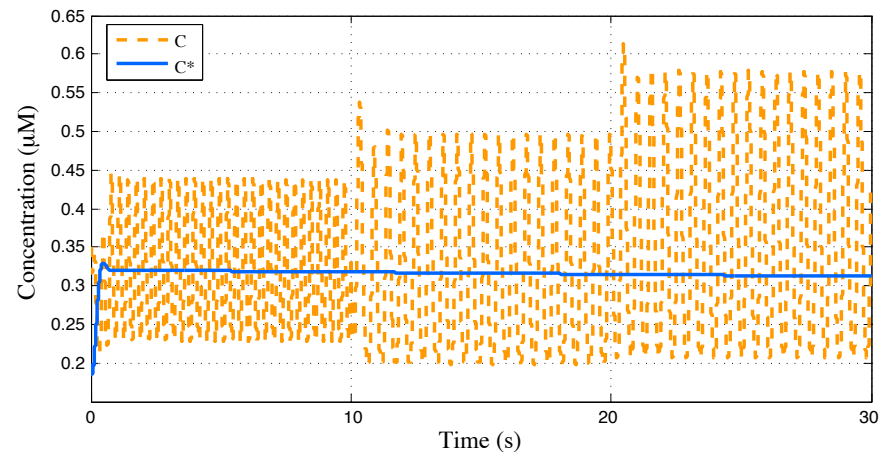

Fig. 10: Elimination of $\mathrm{Ca}^{2+}$ oscillation using the proposed feedback and feed-forward control technique. Regular $\mathrm{Ca}^{2+}$ oscillations $C$ (dashed line) is compared to a controlled $\mathrm{Ca}^{2+}$ level $C^{*}$ (solid line) for $\mathrm{IP}_{3}=0.5(\mu M)$.

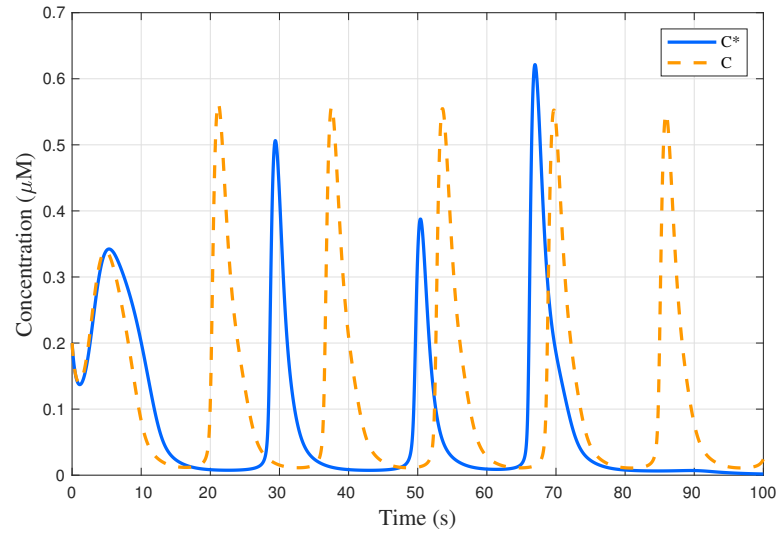

Fig. 11: Regulated oscillatory control of $\mathrm{Ca}^{2+}$ oscillation using the proposed feedback and feed-forward control technique. Irregular $\mathrm{Ca}^{2+}$ oscillations from pre-synaptic influence $C$ (dashed line) is compared to a controlled $\mathrm{Ca}^{2+}$ level $C^{*}$ (solid line) for $\mathrm{IP}_{3}=0.5$ $(\mu M)$.

the approach to stabilising the disturbance. To achieve this, a disturbance component was added to Eq. 1, through addition of Gaussian noise [28]. This additional noise affects the $\mathrm{Ca}^{2+}$ level in the astrocyte cell's cytosol.

As illustrated in Fig. 12, the feed-forward control can maintain the levels of $\mathrm{Ca}^{2+}$ even with the additional Gaussian noise. This is obtained by the relation of the current $\mathrm{Ca}^{2+}$ concentration levels with the desired level, and therefore, this stability is achieved through the adjustment of $\beta$. This demonstrates the excessive control fluctuations in $\mathrm{Ca}^{2+}$ concentration levels from additional noise.

3) Maintaining Stable $\mathrm{Ca}^{2+}$ Concentration: While the previous section presented the case of maintaining stable $\mathrm{Ca}^{2+}$ concentration due to excessive noise, in this section, the impact of overall fluctuations in the cytosolic concentration of the cell is discussed. The ability to maintain the stability of the cytosolic $\mathrm{Ca}^{2+}$ concentration levels in astrocyte cells

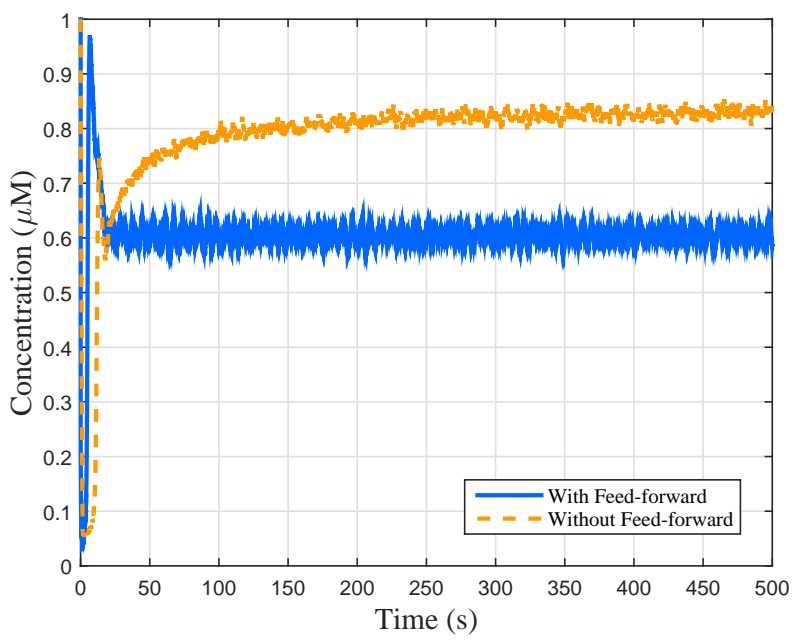

Fig. 12: The application of the Feed-forward technique to maintain the desired levels of $\mathrm{Ca}^{2+}$ in the astrocytes' cytosol. 


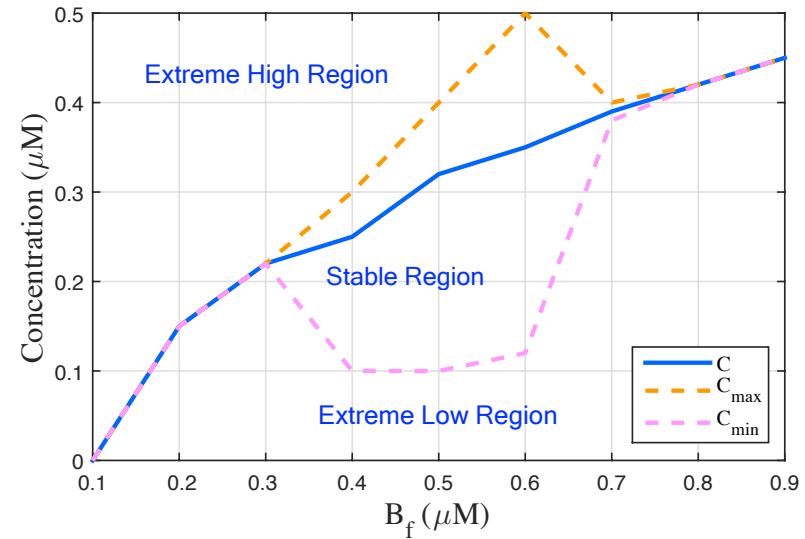

Fig. 13: Application of the control model to achieve the desired $\mathrm{Ca}^{2+}$ level compared to regions that can result in diseases. Extreme high region is any value higher than the maximum $\mathrm{Ca}^{2+}$ levels, Extreme low region is any value lower than the minimum $\mathrm{Ca}^{2+}$ levels, and the stable region is the $\mathrm{Ca}^{2+}$ levels in between the maximum and minimum values.

can lead to not only a healthy state of the cell but also the synaptic transmission quality in the tripartite synapses. In the event of fluctuations (extreme low or high) in the overall concentration, this can lead to some neurodegenerative diseases. For example, low $\mathrm{Ca}^{2+}$ concentration leads to cell death and poor functioning of neurons that cause depression, whereas high $\mathrm{Ca}^{2+}$ concentration is linked with one of the causes of Alzheimer's disease [29], [30], [31]. For evaluation of both extreme high and low concentrations, a simple model is presented that shows the effectiveness of the control technique in maintaining the stable zones of $\mathrm{Ca}^{2+}$ concentration levels. We do not claim that this technique can directly be applied to the treatment of neurodegenerative diseases. This method is used only in micro-levels of the astrocyte communication and tripartite synapses. Since neurodegeneration affects higher levels of communication in the brain, there needs to be an extended version of this system that achieves the very challenging distributed astrocyte control.

There is a variation of $\beta$ in Eq. 3 from 0.1 to $0.9 \mu M$ that is compared with the resulting $\mathrm{Ca}^{2+}$ concentration levels with the feed-forward and feedback control technique. In the case of when no control is applied, $\mathrm{Ca}^{2+}$ oscillations are expected. Based on the oscillations, the maximum and minimum values are selected of the final concentrations and used them to define three regions: extreme high region, extreme low region, and stable region. The extreme high region consists of any value higher than the maximum $\mathrm{Ca}^{2+}$ concentration levels, while the extreme low region contains any value lower than the minimum $\mathrm{Ca}^{2+}$ concentration levels. The stable region, which represents the safe level in the $\mathrm{Ca}^{2+}$ concentration, is in between the extreme limits.

Regulation of $\mathrm{Ca}^{2+}$ concentration can maintain stability in the concentration within the safe region for all $\mathrm{IP}_{3}$ values as illustrated in Fig. 13. The $C_{f}$ from Eq. 12 was selected based on the central value between the maximum and minimum values of $C$. The $\beta_{f}$ was computed based on $C_{f}$, and by adjusting this, the system will change $C$ to match with $C_{f}$. There is a concern about the long-term usage of the proposed technique in regards to cell function homoeostasis. Even though the $\mathrm{Ca}^{2+}$ concentration levels can be maintained for long periods of time (minutes), we do not guarantee that cellular function will not be changed in longer periods. This will require novel protocol solutions that activate and deactivate the control autonomously under certain condition and can be realised through synthetic biology.

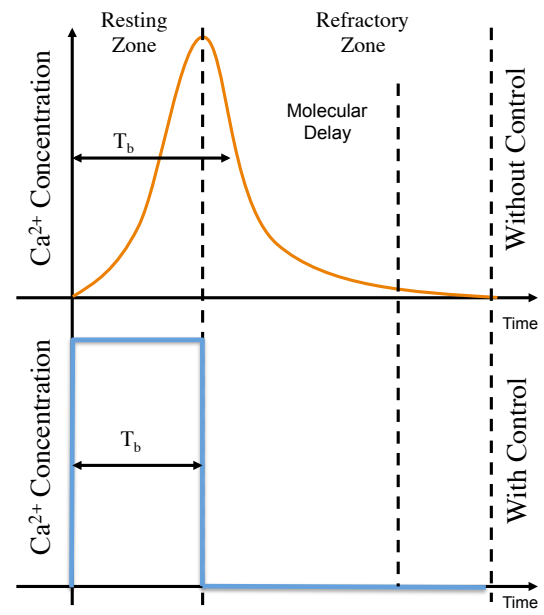

Fig. 14: Elimination of the refractory period of a transmitted signal using the proposed control function.

4) Data Rate: One of the issues regarding the use of $\mathrm{Ca}^{2+}$ signalling for molecular communication is the artificial stimulation of the ions for communication purpose, as well as the excessive noise that can result in poor data rate performance. Barros et. al. [18], [19], showed that large $T_{b}$ is desired to achieve reasonable communication capacity but this results in poor data rate for most types of cells that communicate using $\mathrm{Ca}^{2+}$. Another reason for the long bit transmission periods $T_{b}$ is due to the refractory time of the $\mathrm{Ca}^{2+}$ oscillation. The refractory time is an inherent process found in $\mathrm{Ca}^{2+}$ intracellular signalling, where the concentration of the fluctuating ions are required to stabilise before they can be stimulated again, Fig. 14. The objective now is to integrate the proposed feed-forward and feedback control technique towards the refractory time elimination inside the system equaliser.

To show the benefits of the proposed control method for molecular communication, a data rate analysis is presented of a single hop $\mathrm{Ca}^{2+}$ molecular communication system using astrocytes. The data rate in such system can be computed using: $1 /\left(T_{b} * N b\right)$, where $N b$ is the number of bits transmitted. Three $T_{b}$ values were used $(5 s, 10 s, 50 s)$ and compared with the performance for the case when the system integrates the control model as well as without, and varying $N b$ in the process. As demonstrated in Fig. 15 the elimination of the refractory time provides substantial benefit for improving the data rate for all the $T_{b}$ values. On average, the refractory time takes up to five seconds to be completed. This time is needed for a full oscillation cycle from the signalling process of the cell. However, such oscillatory process can also be eliminated with the proposed feed-forward and feedback control tech- 

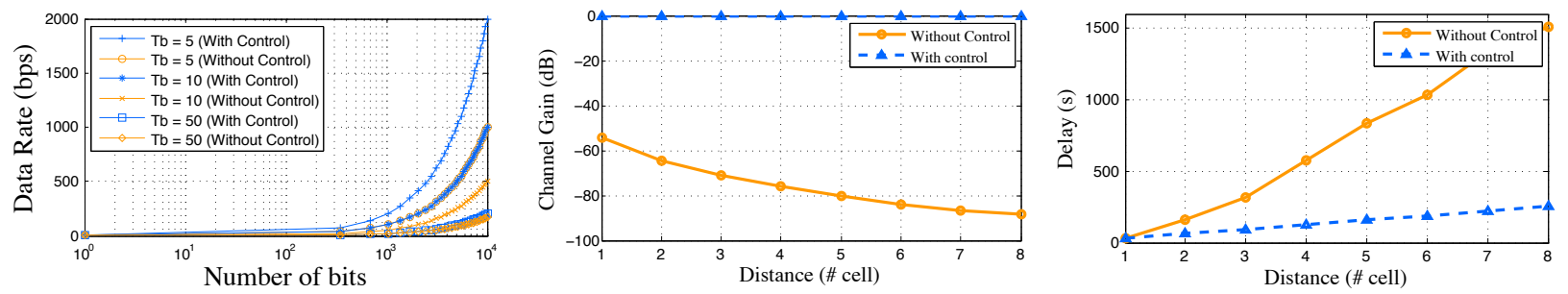

Fig. 15: Data rate performance of a $\mathrm{Ca}^{2+}$. Fig. 16: Gain performance of a $\mathrm{Ca}^{2+}$ - Fig. 17: Delay performance of a $\mathrm{Ca}^{2+}-$ signalling molecular communication sys- signalling molecular communication sys- signalling molecular communication system with $T b$ values $(5 s, 10 s, 50 s)$. Com- tem with $T b$ values $(5 s, 10 s, 50 s)$. Com- tem with $T b$ values $(5 s, 10 s, 50 s)$. Comparison of the performance when the feed-parison the performance when the feed-parison of the performance when the feedforward and feedback control technique is forward and feedback control technique is forward and feedback control technique is applied against the system with no con- applied against the system with no con- applied against the system with no control technique that has to wait for longer trol technique that has to wait for longer trol technique that has to wait for longer refractory periods. refractory periods. refractory periods.

nique achieving higher data rate values. For example, with the highest values of data rate $\left(T_{b}=5 s\right)$, without control reaches a limit of 1000bps while with control reaches 2000bps.

5) Molecular Gain: Due to the equal diffusion direction probability (27), $\mathrm{Ca}^{2+}$ may not reach the $R x$. Naturally, the amplitude of the received signal is negatively affected by longer distances between the $T x$ and $R x$. This phenomenon is analysed using gain, which is calculated using the following formula [19]:

$$
\Gamma(f)=10 \log \left(\frac{\Gamma_{T}(f)}{\Gamma_{T 0}(f)}\right)
$$

where $\Gamma_{T}(f)$ is the average peak concentration and $\Gamma_{T 0}(f)$ is the initial peak concentration.

Ideal control of the received $\mathrm{Ca}^{2+}$ leads to the maintenance of gain performance over distance, Fig. 16. The equaliser provides total recovery in both transmitter and receiver. However, these results might be different for other modulation schemes different than the OOK. On top of that, there must be a distance limit, where signals cannot be reached, and the activation of the control technique cannot be performed.

6) Molecular Delay: To fairly measure the amount of molecules that are transmitted as well as the time it travels over a certain distance of cellular tissue, the molecular delay proposed in [19] is used to capture this phenomenon. In this way, the delay is an appropriately measured for each bit, since the information is encoded into a molecular concentration of $\mathrm{Ca}^{2+}$ signals. The proposed method is modelled by the following formula:

$$
\int_{0}^{T_{R}} C_{R}(x, t) \mathrm{d} t=\int_{0}^{T_{T}} C_{T}(t) \mathrm{d} t
$$

in which, $C_{R}$ is a function that returns the $\mathrm{Ca}^{2+}$ concentration of a $R x$ at time $t, C_{T}$ returns the $T x \mathrm{Ca}^{2+}$ concentration at time $t, T_{T}$ is the time slot length of the $T x$ and $T_{R}$ is the time slot length of the $R x$. Here, the end-to-end delay will be $T_{R}$.

Delay is considerably reduced due to the contribution of the regenerated molecules in the receiver by the feed-forward feedback control technique in the form of the equaliser, Fig. 17. The elimination of the refractory periods is another contributor to the delay performance since the remaining interbit interference is also minimised. The difference of delay compared to gain performance is that delay performance should be similar even with different modulation schemes that keep the signal energy levels the same. This result is significant for the minimisation of distance effects over the delay, decreasing around an average of $60 \%$ delay values per unit distance (number of cells away).

\section{DISCUSSION}

In this section, two main applications are explored for the proposed $\mathrm{Ca}^{2+}$ control method, including prevention of neurodegenerative diseases and speeding up molecular communication. A significant impact on these topics will likely result from the utilisation of the proposed method.

\section{A. Prevention of Neurodegenerative Diseases}

Approximately 24 million people worldwide have dementia or neurodegenerative diseases with an annual cost of about $\$ 226$ billion in the U.S. alone [32], [33]. Causes of such remain unknown, and only conventional symptomatic treatments are available for improving the patients' health. This is achieved with drugs that target the symptoms alone, neither treating the underlying disease or at best delaying its progression. However, as the disease progresses, brain cells die, and connections among cells are lost, causing the disease symptoms to worsen [34].

A major issue with drug delivery techniques to the brain is the numerous protective barriers that encapsulate the central nervous system, and one of this example is the bloodbrain barrier [34]. Overcoming the blood-brain barrier can be achieved through biotechnology, synthetic biology, as well as nanotechnology, and this can lead to efficient and directed therapeutic tools. The control model proposed in this paper can be developed from a combination of nanoparticles and gene therapy that are used to control the $\mathrm{Ca}^{2+}$ signalling, as well as synthetic biology, towards future in-vivo settings [17]. These nanoparticles can be inserted into the brain through minimally invasive surgeries, however, they only achieve single-cell stimulation and their wide deployment in the brain remains unclear. Novel self-assembly nanoparticles showed promising results to bypass the blood-brain barrier and induce wide neuronal response using Gold or Albumin [35], [36], [37]. However, 
in the case of regulating astrocyte intracellular $\mathrm{Ca}^{2+}$ through $\mathrm{IP}_{3}$ control, we need to possibly design these self-assembly nanoparticles based on Carbon Black, Titanium Dioxide or Zinc Oxide [38], [39]. Even though promising, the usage of these nanoparticles increase toxicity levels in the cell, and their in-vivo applicability remains an interesting research challenge [40].

In the case of synthetic biology, programming of cellular signalling pathways can be achieved, leading to stable levels of $\mathrm{Ca}^{2+}$ directly, preventing fluctuations that may result in diseases. This approach would likely try to replicate the oscillatory control results presented in Fig. 11. This is a more complex solution compared to the other approaches mentioned above, especially for gene-therapy in the brain. Alternatively, some works have investigated mechanisms using synthetic biology to engineer neurons to achieve sensitivity to light at a particular wavelength, providing a new alternative for externally controlled neural stimulation, which is termed optogenetics. These reprogrammed genes can be transmitted to the brain based on virus communication to neuronal cells. However, optical cables are used to transmit light into the brain. By using ultrasound communication, the so-called Wireless Optogenetic Nanonetworks is an alternative to eradicate the usage of optical cables [4]. Even though ultrasound transceivers are unstable, they are an alternative to access deeper levels in the brain, and subsequently provide treatment for many neurodegenerative diseases, and in this case, control the cellular signalling. The ultrasound transceiver will control the nanodevices with nanoLED technology to stimulate either neurons or astrocytes directly. The stimulation of neurons directly is not an attractive approach due to the poor conditions of the synaptic quality, which is largely contributed by the astrocytes. The integration of the proposed control theory approach and the wireless optogenetics nanonetworks can potentially contribute to the next generation of brain-machine interfaces.

\section{B. Molecular Communication}

Low data rates are a natural characteristic of molecular communication systems due to many factors including stochastic propagation delay as well as excessive noise [2]. These are due to the natural biological processes that result in poor communication performance. The contribution provided in this paper is far from solving the problem of integrating control theory and synthetic biology with molecular communications, due to the plurality of biological communication channels. These channels are inherently specific with many different characteristics which indicate that control solutions must be specific as well. However, our solution as a promising equalisation technique that is likely to benefit other communication systems in different types of tissues as well. Therefore, more investigation needs to be carried out to counter these natural processes that can affect the communication performance. These solutions will usually come from nanotechnology through components and materials to control the biological process or through manipulation of a cell using techniques from synthetic biology.

In Section VI-4, we show how the proposed control technique can equalise the signal, eliminating the refractory period and increasing the overall performance including data rate, delay and gain improvements. However, our proposed solution is not limited to this particular technique, as it can be modified and applied to other parts of the communication system as well, including in modulation and noise cancellation. Recent work has proposed a modulation technique for a $\mathrm{Ca}^{2+}$-signalling based molecular communication system [41], where digital modulation schemes such as On-Off ShiftKeying $(O O K)$ were used in conjunction with various error control techniques. Our control scheme can be applied to that particular scenario by implementing complex modulation schemes based on multi-level information encoding. The control method for noise cancelling purposes can be engineered into the biological nanomachines that utilise $\mathrm{Ca}^{2+}$-signalling based molecular communication system. In [18], noise in $\mathrm{Ca}^{2+}$-signalling molecular communication was studied and quantified showing that its high concentration can negatively affect the communication system performance. The noise will emanate at the transmitter as the $\mathrm{Ca}^{2+}$ waves are stimulated, along the path as the waves are propagated, and at the receiver, as they stimulate $\mathrm{Ca}^{2+}$ to receive digital bits. Therefore, based on this scenario, and also on our technique by which the effect of noise can be limited, the control model can be engineered into the cells of the tissue to cancel noise as they propagate along the channel. This can be achieved by restricting the quantity of $\mathrm{IP}_{3}$ for each cell that represents the transmitter, along the propagation path, as well as the receiver.

\section{CONCLUSIONS}

In recent years, nanotechnology has brought together some different disciplines including synthetic biology and engineering, where the objective is to develop novel healthcare solutions to detect, prevent and cure diseases. This includes the field of molecular communication, where it aims to model and construct biological communication systems for inter and intracellular cellular signalling. This new area of research seeks to develop new approaches for detecting and preventing diseases that can emerge from impairments in the communication process, as well as create artificial communication processes that connect a network of nanomachines. This paper investigated one particular type of molecular communication that utilises $\mathrm{Ca}^{2+}$ signalling between astrocyte cells and pre and post-synaptic neurons. This three-way communication process is known as the tripartite synapse. In particular, the focus is on the application of a feed-forward and feedback control technique to maintain the stability of $\mathrm{Ca}^{2+}$ levels as intercellular signalling is conducted between the cells. The application of the control model achieved firstly, tight regulation of $\mathrm{Ca}^{2+}$ concentration, demonstrably maintaining a stable level to minimise any fluctuations, and secondly, an improvement of the overall performance in molecular communication using $\mathrm{Ca}^{2+}$-signalling in astrocyte cells. Previous studies have shown that $\mathrm{Ca}^{2+}$-signalling in cellular tissue can lead to a significant quantity of noise within the environment, impairing the overall system performance. However, applying the control model has resulted in the reduction in the refractory period of the $\mathrm{Ca}^{2+}$-signalling leading to smaller time-slots for bit transmissions, and higher data rates. The control model proposed 
in this paper can pave the way for novel techniques for disease prevention, as well as mechanisms to improve the performance of molecular communication systems. Furthermore, future work will be carried out to investigate improvements in neural activity by enhancing glutamate propagation through control of astrocyte $\mathrm{Ca}^{2+}$ signalling and regulating the $\mathrm{IP}_{3}$ dynamics so that it does not interfere with the synchronization between the pre-synaptic neuron and the astrocytes and potentially decrease communication errors in the synaptic channel.

\section{REFERENCES}

[1] B. Atakan, S. Balasubramaniam, and O. B. Akan, "Body area nanonetworks with molecular communications in nanomedicine," IEEE Communications Magazine, vol. 50, pp. 28-34, 2012.

[2] M. T. Barros, "Ca2+-signaling-based molecular communication systems: Design and future research directions," Nano Communication Networks, vol. 11, no. 1, pp. 103-113, 2017.

[3] I. Akyildiz, M. Pierobon, S. Balasubramaniam, and Y. Koucheryavy, "The internet of bio-nano things," IEEE Communications Magazine, vol. 53 , no. 3, pp. 32-40, March 2015.

[4] S. A. Wirdatmadja, M. T. Barros, Y. Koucheryavy, J. M. Jornet, and S. Balasubramaniam, "Wireless optogenetic nanonetworks for brain stimulation: Device model and charging protocols," IEEE Transactions on NanoBioscience, vol. 16, no. 8, pp. 859-872, Dec 2017.

[5] I. Akyildiz, F. Fekri, R. S. C. R. Forest, and B. K. Hammer, "Monaco: fundamentals of molecular nano-communication networks," IEEE Wireless Communications, vol. 19, no. 5, pp. 12-18, 2012.

[6] M. Pierobon and I. F. Akyildiz, "Capacity of a diffusion-based molecular communication system with channel memory and molecular noise," IEEE Transactions on Information Theory, vol. 59, no. 2, pp. 942-954, 2013.

[7] F. Mesiti, M. Veletć, P. A. Floor, and I. Balasingham, "Astrocyte-neuron communication as cascate of equivalent circuits," Nano Communication Networks, 2015

[8] E. A. Newman, "New roles for astrocytes: Regulation of synaptic transmission," Trends in Neuronscience, vol. 36, no. 10, pp. 536-542, 2003.

[9] S. Nadkarni and P. Jung, "Dressed neurons: modeling neural-glial interactions," Physical Biology, vol. 1, no. 1, p. 35, 2004.

[10] Q.-S. Liu, Q. Xu, J. Kang, and M. Nedergaard, "Astrocyte activation of presynaptic metabotropic glutamate receptors modulates hippocampal inhibitory synaptic transmission," Neuron Glia Biology, vol. 1, pp. 307316, 112004.

[11] C. Luscher and R. C. Malenka, "Nmda receptor-dependent long-term potentiation and long-term depression (ltp/ltd)," Cold Spring Harbor Perspectives in Biology, vol. 4, no. 6, p. a005710, 2012.

[12] M. M. Halassa, T. Fellin, and P. G. Haydon, "The tripartite synapse: roles for gliotransmission in health and disease," Trends in Molecular Medicine, vol. 13, no. 2, pp. 54 - 63, 2007.

[13] G. Perea, M. Navarrete, and A. Araque, "Tripartite synapses: astrocytes process and control synaptic information," Trends in Neurosciences, vol. 32, no. 8, pp. $421-431,2009$.

[14] P. Raul, S. Manyam, P. Pagilla, and S. Darbha, "Output regulation of nonlinear systems with application to roll-to-roll manufacturing systems," IEEE/ASME Transactions on Mechatronics, vol. 20, no. 3, June 2015.

[15] K. Le Meur, J. Mendizabal-Zubiaga, P. Grandes, and E. Audinat, "Gaba release by hippocampal astrocytes," Frontiers in computational neuroscience, vol. 6, 2012.

[16] M. Pitta, M. Goldberg, V. Volman, H. Berry, and E. Ben-Jacob, "Glutamate regulation of calcium and ip3 oscillating and pulsating dynamics in astrocytes," Journal of Biological Physics, vol. 35, pp. 383-411, 2009.

[17] M. T. Barros and S. Dey, "Set point regulation of astrocyte intracellular ca2+ signalling," in The 17th IEEE International Conference on Nanotechnology (IEEE NANO 2017),, 2017, pp. 315-320.

[18] M. T. Barros, S. Balasubramaniam, B. Jennings, and Y. Koucheryavy, "Transmission protocols for calcium signaling based molecular communications in deformable cellular tissues," IEEE Transactions on Nanotechnology, vol. 13, no. 4, pp. 779-788, 2014.

[19] M. T. Barros, S. Balasubramaniam, and B. Jennings, "Comparative endto-end analysis of ca2+ signaling-based molecular communication in biological tissues," IEEE Transactions on Communications, vol. 63 no. 12 , pp. 5128-5142, December 2015.
[20] - "Using information metrics and molecular communication to detect cellular tissue deformation," IEEE Transactions on Nanobioscience, vol. 13, no. 3, pp. 278-288, 2014.

[21] J. Lallouette, M. D. Pitta, E. Ben-Jacob, and H. Berry, "Sparse shortdistance connection enhance calcium wave propagation in a 3D model of astrocytes networks," Frontiers in Computation Neuroscience, vol. 8 , no. 45, pp. 1-18, 2014.

[22] D. T. Gillespie, "Exact stochastic simulation of coupled chemical reactions," Journal of Physical Chemistry, vol. 81, no. 25, pp. 23402361, 1977.

[23] D. Kilinc and O. B. Akan, "An information theoretical analysis of nanoscale molecular gap junction communication channel between cardiomyocytes," IEEE Transactions on Nanotechnology, vol. 12, no. 2, pp. 129-136, March 2013.

[24] I. Llatser, A. Cabellos-Aparicio, M. Pierobon, and E. Alarcon, "Detection techniques for diffusion-based molecular communication," IEEE Journal on Selected Areas in Communications, vol. 31, no. 12, pp. 726734, 2013.

[25] T. S. Gardner, C. R. Cantor, and J. J. Collins, "Construction of a genetic toggle switch in escherichia coli," Nature, vol. 403, no. 6767, pp. 339342,2000 .

[26] M. Lavrentovich and S. Hemkin, "A mathematical model of spontaneous calcium(ii) oscillations in astrocytes," Journal of Theoretical Biology, vol. 251, pp. 553-560, 2008.

[27] S. Nadkarni and P. Jung, "Modeling synaptic transmission of the tripartite synapse," Physical biology, vol. 4, no. 1, p. 1, 2007.

[28] R. Janicek, M. Hotka, J. A. Zahradnikova, A. Zahradnikova, and I. Zahradnak, "Quantitative analysis of calcium spikes in noisy fluorescent background," PLOS ONE, vol. 8, no. 5, p. e64394, 052013.

[29] A. Gaur, A. Midha, and A. L. Mbatia, "Applications of nanotechnology in medical sciences," Asian Journal of Pharmaceutical Sciences, vol. 2 , pp. 80-85, 2008.

[30] S. Orrenius and P. Nicotera, "The calcium ion and cell death," Journal of Neural Transmission. Supplementa, vol. 43, pp. 1-11, 1994.

[31] F. M. LaFerla, "Calcium dyshomeostasis and intracellular signalling in alzheimer's disease," Nature Reviews Neuroscience, vol. 3, pp. 862-872, 2002.

[32] A. Association, "Alzheimer's disease facts and figures." [Online]. Available: https://www.alz.org/facts/downloads/facts_figures_2015.pdf

[33] C. P. Ferri, M. Prince, C. Brayne, and et al., "Global prevalence of dementia: A delphi consensus study," Lancet, vol. 366, pp. 2112-2117, 2005.

[34] G. Modi, V. Pillay, and Y. E. Choonara, "Advances in the treatment of neurodegenerative disorders employing nanotechnology," Annals of the New York Academy of Sciences, vol. 1184, pp. 154-172, 2010.

[35] D. Lovisolo, A. Gilardino, and F. A. Ruffinatti, "When neurons encounter nanoobjects: spotlight on calcium signalling," International journal of environmental research and public health, vol. 11, no. 9, pp. 9621-9637, 2014.

[36] Q. Feng, Y. Shen, Y. Fu, M. E. Muroski, P. Zhang, Q. Wang, C. Xu, M. S. Lesniak, G. Li, and Y. Cheng, "Self-assembly of gold nanoparticles shows microenvironment-mediated dynamic switching and enhanced brain tumor targeting," Theranostics, vol. 7, no. 7, p. 1875, 2017.

[37] T. Lin, P. Zhao, Y. Jiang, Y. Tang, H. Jin, Z. Pan, H. He, V. C. Yang, and Y. Huang, "Blood-brain-barrier-penetrating albumin nanoparticles for biomimetic drug delivery via albumin-binding protein pathways for antiglioma therapy," ACS nano, vol. 10, no. 11, pp. 9999-10 012, 2016.

[38] S. Hussain, L. C. Thomassen, I. Ferecatu, M.-C. Borot, K. Andreau, J. A. Martens, J. Fleury, A. Baeza-Squiban, F. Marano, and S. Boland, "Carbon black and titanium dioxide nanoparticles elicit distinct apoptotic pathways in bronchial epithelial cells," Particle and fibre toxicology, vol. 7 , no. 1, p. 10,2010

[39] A. Gilardino, F. Catalano, F. A. Ruffinatti, G. Alberto, B. Nilius, S. Antoniotti, G. Martra, and D. Lovisolo, "Interaction of sio2 nanoparticles with neuronal cells: Ionic mechanisms involved in the perturbation of calcium homeostasis," The international journal of biochemistry \& cell biology, vol. 66, pp. 101-111, 2015.

[40] D. Lovisolo, M. Dionisi, F. A. Ruffinatti, and C. Distasi, "Nanoparticles and potential neurotoxicity: focus on molecular mechanisms," 2018.

[41] M. T. Barros, S. Balasubramaniam, and B. Jennings, "Error control for calcium signaling based molecular communications," in 47th Annual Asilomar Conference on Signals, Systems, and Computers, 2013. 


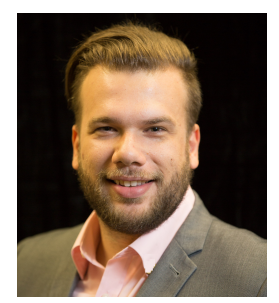

Michael Taynnan Barros was born in Campina Grande, Brazil, 1990. He is currently an Irish Research Council Government of Ireland Postdoctoral research fellow associated with the TSSG, WIT. Michael received his $\mathrm{PhD}$ in Telecommunication Software at the Waterford Institute of Technology in 2016, M.Sc. degree in Computer Science at the Federal University of Campina Grande in 2012 and B.Tech. degree in Telematics at the Federal Institute of Education, Science and Technology of Paraiba in 2011. He has published over 40 research papers in diverse journals such as IEEE Transactions on Communications, IEEE Transactions on Nanotechnology, and conferences in the area of wireless communications, optical communications, ad-hoc networks, as well as molecular and nanoscale communications. He is also a reviewer for many journals and participated as technical program committee and reviewer for various international conferences. In 2017, he served as the be the Technical Program Co-chair for the 3rd International Workshop on Nanoscale Computing and Communications (NsCC) held in conjunction with NEW2AN conference, the chair of the 5GPPP Network Management, QoS and Security Working Group and the Chair of the 2nd Network Management, QoS and Security for 5G Networks held in conjunction with the EuCNC. Interests in Molecular Communications, Nanonetworks and 5G Technology for Connected Health.

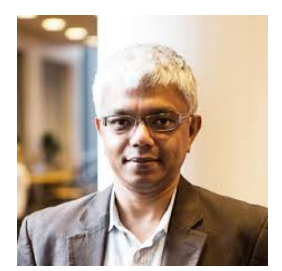

Subhrakanti Dey (M'96 - SM'06) was born in India. He received the B.Tech. and M.Tech. degrees from the Department of Electronics and Electrical Communication Engineering, Indian Institute of Technology, Kharagpur, in 1991 and 1993, respectively, and the Ph.D. degree from the Department of Systems Engineering, Research School of Information Sciences and Engineering, Australian National University, Canberra, in 1996. e is currently a Professor with the Institute of Telecommunications Research (ITR), University of South Australia, Adelaide. Prior to this, he was the Professor of Wireless Sensor Networks with Dept of Engineering Sciences in Uppsala University, Sweden during 2013-2017, and a Professor with the Department of Electrical and Electronic Engineering, University of Melbourne, Parkville, Australia, from 2000 until early 2013. From September 1995 to September 1997, and September 1998 to February 2000, he was a Postdoctoral Research Fellow with the Department of Systems Engineering, Australian National University. From September 1997 to September 1998, he was a Postdoctoral Research Associate with the Institute for Systems Research, University of Maryland, College Park. His current research interests include networked control systems, wireless communications and networks, signal processing for sensor networks, and stochastic and adaptive estimation and control. Professor Dey currently serves on the Editorial Board of the IEEE Transactions on Signal Processing, IEEE Transactions on Control of Network Systems and IEEE Transactions on Wireless Communications. He was also an Associate Editor for the IEEE Transactions On Signal Processing during 2007-2010 and the IEEE Transactions On Automatic Control during 2004-2007. 